\title{
MODELLING OF KINETICS AND DILATOMETRIC BEHAVIOR OF NON-ISOTHERMAL PEARLITE-TO-AUSTENITE TRANSFORMATION IN AN EUTECTOID STEEL.
}

\author{
C. García de Andrés ${ }^{1}$, F.G. Caballero ${ }^{1,2}$,C. Capdevila ${ }^{1}$ and H.K.D.H. Bhadeshia ${ }^{2}$ \\ ${ }^{1}$ Department of Physical Metallurgy \\ Centro Nacional de Investigaciones Metalúrgicas (CENIM) \\ Consejo Superior de Investigaciones Científicas (CSIC) \\ Avda. Gregorio del Amo, 8. 28040 Madrid, Spain \\ ${ }^{2}$ Department of Materials Science and Metallurgy \\ University of Cambridge \\ Pembroke Street, Cambridge CB2 3QZ, UK
}

\section{Introduction}

Austenitization is an inevitable occurrence during the heat treatment of steels. Despite this consideration, less attention has been paid so far to the study of the formation of austenite as compared with the vast amount of research on its decomposition. That is because the steel properties depend basically on the transformation processes following austenitization. However, the initial austenitic condition is important to the development of the final microstructure and its mechanical properties. In this sense, continuous heating transformation diagrams (CHT) of different martensitic stainless steels and low carbon microalloyed steels were studied in previous work (1-3). With the introduction of dual-phase steels (4-7), partial austenitization in the intercritical temperature region became of technological interest and was widely studied (8-11). In that work, the authors emphasise the importance of the microstructure immediately before intercritical annealing. Roosz et al. (12) determined the influence of the initial microstructure on the nucleation rate and grain growth of austenite during isothermal treatment of a eutectoid plain carbon steel.

The Avrami equation is generally used to model transformations under isothermal conditions (13). However, in this paper we present a model in which this equation has been applied successfully in the modelling of the pearlite-to-austenite transformation during continuous heating in a eutectoid steel with a fully pearlitic initial microstructure. Moreover, since dilatometric analysis is a technique very often employed to study phase transformations in steels, calculations of relative change in length have been made as function of temperature, and the differences between theoretical and experimental results have been analysed.

\section{Material and experimental procedure}

A eutectoid carbon steel with the composition of C 0.76, Si 0.24, Mn 0.91, P 0.013 wt.\%, was used. The following heat treatment was carried out to make sure that the microstructure of this steel is fully pearlitic. Specimens were austenized at $1273 \mathrm{~K}$ for $5 \mathrm{~min}$, quenched to the isothermal pearlite transformation temperature of $923 \mathrm{~K}$ and then, after a holding time of $10 \mathrm{~min}$, were cooled rapidly to room temperature. Specimens were polished in the usual way and finished on $0.25 \mu \mathrm{m}$ diamond paste for metallographic examination. An etching solution of picric acid in isopropyl alcohol with several drops of Vilella's reagent was used to disclose the pearlite morphology on a Jeol JXA-820 scanning electron microscope (Fig. 1).

TABLE 1

Morphological parameters for pearlite

\begin{tabular}{ccc}
\hline$\sigma_{o} \times 10^{-4}, \mathrm{~mm}$ & $S_{v}{ }^{P P}, \mathrm{~mm}^{-1}$ & $a^{P} \times 10^{-3}, \mathrm{~mm}$ \\
\hline $0.9 \pm 0.2$ & $1295 \pm 283$ & $1.9 \pm 0.4$ \\
\hline
\end{tabular}

Two characteristic morphological parameters of the fully pearlitic microstructure were determined: the average true interlamellar spacing $\left(\sigma_{o}\right)$ and the specific interface of the pearlite colonies $\left(S_{v}{ }^{P P}\right)$. The value of $\sigma_{o}$ was obtained from electron micrographs according to Underwood's intersection procedure (14). The specific interface of the pearlite colonies was measured as reported by Roosz et al. (12). Approximating the pearlite colony with a truncated octahedron, the edge length of the pearlite colonies $\left(a^{P}\right)$ is: 
$a^{P}=\frac{2.367}{S_{v}^{P P}} \quad \mathrm{~mm}$

The data for $\sigma_{o}, S_{v}{ }^{P}$ and $a^{P}$ are shown in Table 1.

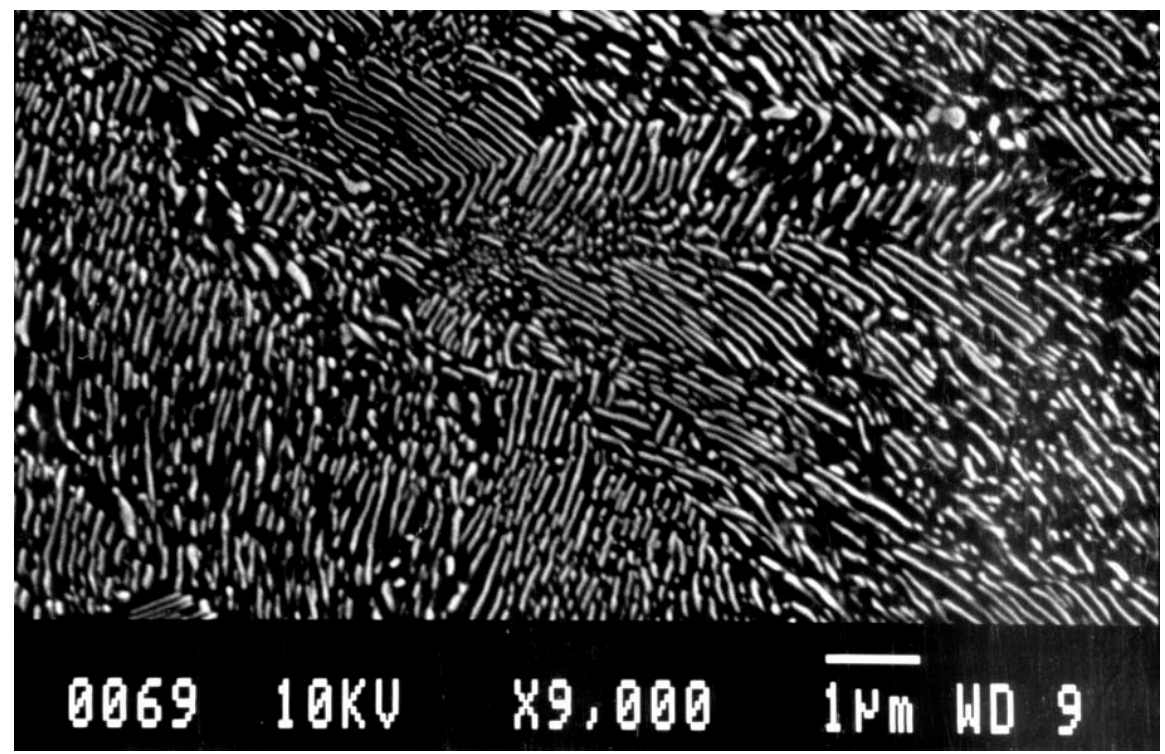

Figure 1. SEM micrographs of the steel after isothermal heat treatment at $923 \mathrm{~K}$ for $10 \mathrm{~min}$. Picric Acid in isopropyl alcohol.

To validate the austenitization model and the calculated dilatation curve, a dilatometric specimen 3 mm thick and $12 \mathrm{~mm}$ long was heated at a constant rate of $0.05 \mathrm{~K} / \mathrm{s}$ in a vacuum of $1 \mathrm{~Pa}$. For this purpose an Adamel Lhomargy DT1000 high-resolution dilatometer was used.

\section{$\underline{\text { Results and discussion }}$}

\section{Modelling of non-isothermal austenite formation kinetics}

Processes including nucleation and growth under isothermal condition can be described in general with Avrami equation (15):

$x=1-\exp \left(-K t^{n}\right)$

where $x$ represents the transformed volume fraction, $K$ is a constant for a given temperature, $t$ is the time and $n$ is a constant characterising the kinetics. To consider a value of $n=4$ means that the nucleation rate ( $\dot{N}$ ) and the growth rate $(G)$ are constant in time (16) and no saturation occurs during the nucleation process (12). For spherical particles this results in a transformed volume fraction of:

$x=1-\exp \left(-\frac{\pi}{3} \dot{N} G^{3} t^{4}\right)$

Roosz et al. (12) determined the temperature and structure dependence of $\dot{N}$ and $G$ as a function of the reciprocal value of overheating $\left(\Delta T=T-A c_{1}\right)$,

$$
\dot{N}=\frac{1.378 \times 10^{-12}}{\left[\left(a^{p}\right)^{2} \sigma_{o}\right]^{2}} \exp \left(\frac{-25.38}{T-A c_{1}}\right) \quad \frac{1}{\mathrm{~mm}^{3} \mathrm{~s}}
$$


$G=\frac{7.0 \times 10^{-11}}{\sigma_{o}{ }^{2}} \exp \left(\frac{-29.7}{T-A c_{1}}\right) \quad \frac{\mathrm{mm}}{\mathrm{s}}$

where $T$ is the absolute temperature and $A c_{1}$ is the eutectoid temperature in $\mathrm{K}$.

With the aim of adapting the Avrami equation to non-isothermal conditions, we have taken logarithms and then differentiated equation [3] to calculate the volume fraction of austenite formed during continuous heating at a given temperature:

$d\left(\ln \frac{1}{1-x}\right)=\frac{d x}{1-x}=4 K t^{3} d t$

If we consider a single and constant rate ( $\dot{T}$ ) for the heating condition, time can be expressed as follows,

$d t=\frac{d T}{\dot{T}} \quad t=\frac{\Delta T}{\dot{T}}$

and substituting into equation [6]

$\frac{d x}{1-x}=4 K \frac{\Delta T^{3}}{\dot{T}^{4}} d T$

Integrating in equation $[8]$ in $[0, x]$ and $\left[A c_{1}, T\right]$ intervals on the left and right sides, respectively,

$\int_{0}^{x} \frac{d x}{1-x}=\int_{A c 1}^{T} 4 K \Delta T^{3} \frac{d T}{\dot{T}^{4}}=\int_{A c 1}^{T} \frac{4 \pi}{3 \dot{T}^{4}} \dot{N} G^{3} \Delta T^{3} d T$

it can be concluded that

$x=1-\exp \int_{A c 1}^{T} \frac{4 \pi}{3 \dot{T}^{4}} \dot{N} G^{3} \Delta T^{3}$

where $\dot{N}$ and $G$ are function of temperature. The integral within the exponential was evaluated numerically. The eutectoid temperature $\mathrm{Ac}_{1}$ of the steel was obtained using Andrews' formula (17).

\section{Model of dilatometric behavior during continuous heating}

Assuming that the sample expands isotropically, the change of the sample length $\Delta L$ referred to the initial length $L_{o}$ at room temperature is related to volume change $\Delta V$ and initial volume $V_{o}$ at room temperature for small changes as follows:

$\frac{\Delta L}{L_{O}}=\frac{V-V_{O}}{3 V_{O}}$

Therefore, $\frac{\Delta L}{L_{O}}$ can be calculated from the volumes of the unit cells and the volume fractions of the different phases present at every temperature during continuous heating: 
$\frac{\Delta L}{L_{o}}=\frac{1}{3}\left[\frac{\left(\begin{array}{llllll}2 V_{\alpha} & a_{\alpha}^{3}+\frac{1}{3} V_{\theta} & a_{\theta} & b_{\theta} & c_{\theta}+V_{\gamma} & a_{\gamma}^{3}\end{array}\right)-\left(2 V_{\alpha_{o}} a_{\alpha_{o}}^{3}+\frac{1}{3} V_{\theta_{O}} a_{\theta_{O}} b_{\theta_{O}} c_{\theta_{O}}\right)}{\left(2 V_{\alpha_{O}} a_{\alpha_{O}}^{3}+\frac{1}{3} V_{\theta_{O}} a_{\theta_{O}} b_{\theta_{O}} c_{\theta_{o}}\right)}\right]$

with

$$
\begin{aligned}
& a_{\alpha}=a_{\alpha_{O}}\left[\begin{array}{ll}
1+\beta_{\alpha} & (T-300)
\end{array}\right] \\
& a_{\gamma}=a_{\gamma_{o}}\left[1+\beta_{\gamma}(T-300)\right] \\
& a_{\theta}=a_{\theta_{O}}\left[1+\beta_{\theta}(T-300)\right] \\
& b_{\theta}=b_{\theta_{O}}\left[1+\beta_{\theta}(T-300)\right] \\
& c_{\theta}=c_{\theta_{O}}\left[1+\beta_{\theta}(T-300)\right]
\end{aligned}
$$

where $V_{\alpha_{O}, \theta_{O}}$ are the initial volume fractions of ferrite and cementite respectively at room temperature. $V_{\alpha, \theta, \gamma}$ are the volume fractions of ferrite, cementite and austenite respectively at any transformation temperature. $a_{\alpha_{O}}$ is the lattice parameter of ferrite at room temperature, and $a_{\alpha}$ is the lattice parameter of ferrite at any transformation temperature. The ferrite lattice parameter was taken to be that of pure iron, $a_{\alpha_{O}}=2.866 \mathrm{~A} . a_{\theta_{O}}, b_{\theta_{O}}, c_{\theta_{O}}$ are the lattice parameters of cementite at room temperature, given by 4.5246, 5.0885 and $6.7423 \mathrm{~A}$, respectively (18), and $a_{\theta}, b_{\theta}, c_{\theta}$ are the lattice parameters of cementite at any transformation temperature. $a_{\gamma_{O}}$ is the lattice parameter of austenite at room temperature as a function of the chemical composition of the austenite, and $a_{\gamma}$ is the lattice parameter of austenite at any transformation temperature. $\beta_{\alpha, \theta, \gamma}$ are the linear thermal expansion coefficients of ferrite, cementite and austenite, respectively, in $\mathrm{K}^{-1}$.

The factors of 2 and 1/3 in the numerator of equation [12] are due to the fact that, the unit cell of ferrite and cementite contain 2 and 12 iron atoms respectively, whereas that of austenite has 4 atoms. The volume fractions of ferrite and of cementite in pearlite were considered to be 0.88 and 0.12 , respectively. The austenite volume fraction was calculated at every temperature using equation [10]. The dependence of the lattice parameter of austenite on alloying elements was as reported by Ridley et al. (19) and Dyson and Holmes (20),

$a_{\gamma_{O}}=3.573+0.033 \mathrm{C}+0.00095 \mathrm{Mn}-0.0002 \mathrm{Ni}+0.0006 \mathrm{Cr}+0.0031 \mathrm{Mo}+0.0018 \mathrm{~V}$

where the chemical composition is measured in wt\% and $a_{\gamma_{O}}$ is in $\mathrm{A}$.

The values of the linear thermal expansion of ferrite and austenite considered in these calculations were $\beta_{\alpha}=1.244 \times 10^{-5} \mathrm{~K}^{-1}$ and $\beta_{\gamma}=2.065 \times 10^{-5} \mathrm{~K}^{-1}$ (21). Moreover, the thermal expansion coefficient of cementite increases with temperature (18). Using data published by Stuart and Ridley (18), the expression of the linear expansion coefficient as a function of temperature is:

$\beta_{\theta}=6.0 \times 10^{-6}+3.0 \times 10^{-9}(T-273)+1.0 \times 10^{-11}(T-273)^{2}$

where $T$ is the temperature in $\mathrm{K}$. 


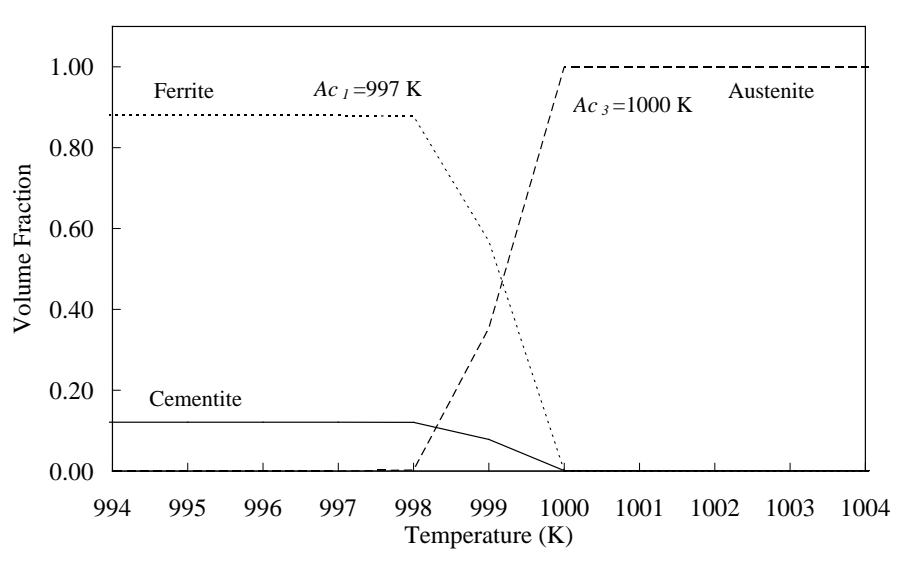

Figure 2. Volume fraction calculated as a function of temperature.

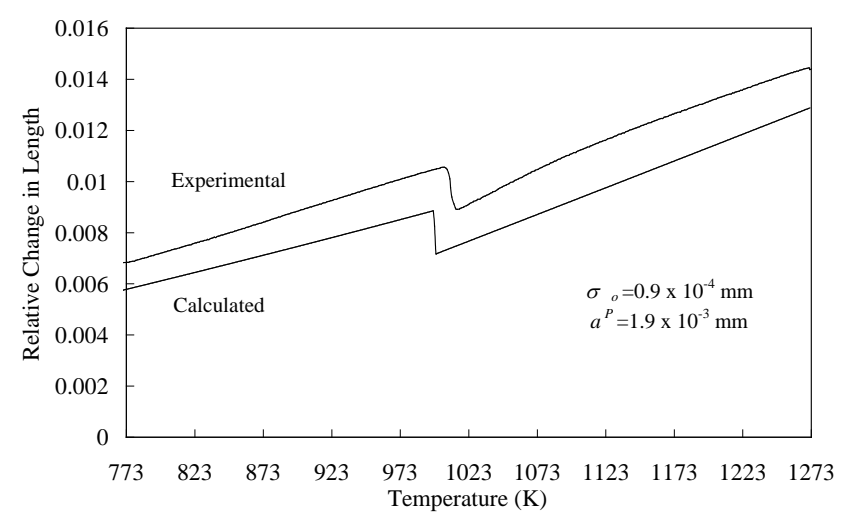

Figure 3. Calculated dilatation curve of an eutectoid steel compared with the experimental curve obtained at a heating rate of $0.05 \mathrm{~K} / \mathrm{s}$.

The calculated austenite formation kinetics and phase fractions are plotted as a function of temperature in Fig. 2. From this diagram it can be seen that the eutectoid reaction ferrite+cementite $\rightarrow$ austenite proceeds within a narrow temperature range. Experimentally, this transformation needs less than $10 \mathrm{~K}$ to reach completion at a heating rate of $0.05 \mathrm{~K} / \mathrm{s}$.

The dilatation curve calculated using equation [12] for a steel with a fully pearlitic initial microstructure and heated at a rate of $0.05 \mathrm{~K} / \mathrm{s}$ is shown in Fig. 3 in comparison with the experimental result. There are three stages in which these dilatation curves could be divided: a) from room temperature to the $\mathrm{Ac}_{1}$ temperature at which pearlite dissolution starts, b) from $\mathrm{Ac}_{1}$ to the $\mathrm{Ac}_{3}$ temperature at which the ferrite+cementite $\rightarrow$ austenite transformation finishes, and, c) from $\mathrm{Ac}_{3}$ to the austenitization temperature at which non-isothermal heating finishes.

The calculated relative change in length was consistent with the measured value at every temperature, although slightly lower. This could be caused by the use of approximate lattice parameters. The calculated linear expansion coefficients of pearlite and austenite are also consistent with those measured coefficients. Experimental kinetic transformation, experimental temperatures of the critical points $\mathrm{Ac}_{1}$ and $\mathrm{Ac}_{3}$ as well as the magnitude of the overall contraction due to austenite formation are accurately reproduced by the calculated dilatation curve.

\section{Conclusions}

1. A mathematical model applying the Avrami equation has been successfully used to reproduce the kinetics of the pearlite-to-austenite transformation in a eutectoid steel during continuous heating. The model used the nucleation and growth functions published by Roosz et al.

2. A model of dilatometric behavior has also been proposed in this work. Experimental dilatometric curve during continuous heating is accurately reproduced by this model.

3. The pearlite-to-austenite transformation proceeds within a narrow temperature range during heating at $0.05 \mathrm{~K} / \mathrm{s}$ in a eutectoid steel. Experimental critical points $\mathrm{Ac}_{1}$ and $\mathrm{Ac}_{3}$ and overall contraction due to austenite formation are in good agreement with the predicted results from both models.

\section{Acknowledgements}

CENIM's authors acknowledge financial support from the European Coal and Steel Community (ECSC-7210. EC/939) and the Spanish Comisión Interministerial de Ciencia y Tecnología (CICYT-MAT95-1192-CE).

\section{$\underline{\text { References }}$}

1. C. García de Andrés, L.F. Alvarez and M. Carsí, Welding International, 6, 612 (1992).

2. L.F. Alvarez and C. García, Mem. Etud. Sci. Rev. Met. 90, 245 (1993). 
3. C. García de Andrés, F.G. Caballero and C. Capdevila, European Coal and Steel Community Final Report (ECSC) Contract No. 7210.EC/807, p. A1-A11, British Steel Technical, Swinden Laboratories, Rotherham (1997).

4. G. Krauss, Steels: Heat Treatment and Processing Principles, p. 274, ASM International, Ohio (1989).

5. A. Gustavsson, D.L. Mcdowell, A. Melander, and M. Larsson, Inst. Metallforsk. Forsk. Rapp. 88, 3145 (1994).

6. V.L. Gadgeel, Tool Alloy Steels, 28, 17 (1994).

7. R.W.K. Honeycombe, Advances in the Physical Metallurgy and Applications of Steels. Proceedings of the International Conference organized by the Metals Society and the University of Liverpool on 21-24 September 1981, p. 38, Metals Society, Liverpool (1981).

8. C.I. García and A.J. DeArdo, Met. Trans. A 12A, 274 (1981).

9. G.R. Speich, V.A. Demarest and R. L. Miller, Met. Trans. A 12A, 1419 (1981).

10. M.M. Souza, J.R.C. Guimaraes and K.K. Chawla, Met. Trans. A 13A, 575 (1982).

11. Xue-Ling Cai, A.J. Garrat-Reed and W.S. Owen, Met. Trans. A 16A, 543 (1985).

12. A. Roósz, Z. Gácsi, and E.G. Fuchs, Acta. Metall. 31, 509 (1983).

13. S. Denis, D. Farias and A. Simon, ISIJ International, 32, 316 (1992).

14. E.E. Underwood, Quantitative Stereology, p. 410, Addison-Wesley Publishing Co., Reading (1970).

15. M. Avrami, J. Chem. Phys. 8, 212 (1940).

16. J.W. Christian, The Theory of Transformations in Metals and Alloys, p. 19, Pergamon Press, Oxford (1965).

17. K.W. Andrews. JISI, 203, 721 (1965).

18. H. Stuart and N, Ridley, JISI, 204, 711 (1966).

19. N. Ridley, H. Stuart, and L. Zwell, Trans. of A.I.M.E, 245, 1834 (1969).

20. D.J. Dyson and B. Holmes, JISI, 208, 469 (1970).

21. M. Takahashi, PhD Thesis, University Cambridge, Cambridge (1992). 